2006

\title{
Determination of rotational and vibrational temperatures of a nitrogen helicon plasma
}

Costel Biloiu

Xuan Sun

Zane Harvey

Earl Scime

Follow this and additional works at: https://researchrepository.wvu.edu/faculty_publications

\section{Digital Commons Citation}

Biloiu, Costel; Sun, Xuan; Harvey, Zane; and Scime, Earl, "Determination of rotational and vibrational temperatures of a nitrogen helicon plasma" (2006). Faculty Scholarship. 124.

https://researchrepository.wvu.edu/faculty_publications/124 


\title{
Determination of rotational and vibrational temperatures of a nitrogen helicon plasma
}

\author{
Costel Biloiu, a) Xuan Sun, Zane Harvey, and Earl Scime \\ Physics Department, West Virginia University, Morgantown, West Virginia 26506
}

(Received 1 May 2006; presented on 10 May 2006; accepted 28 May 2006; published online 5 October 2006)

\begin{abstract}
We present a method of rotational and vibrational temperature estimation in nitrogen plasma by simulation of the emission spectrum of the first positive system $\left(B^{3} \Pi_{g} \rightarrow A^{3} \Sigma_{u}^{+}\right)$of nitrogen. The gas kinetic temperature, assumed to be in equilibrium with the rotational temperature, was obtained from a fit to the experimentally obtained $0 \rightarrow 0$ at $1051 \mathrm{~nm}, 1 \rightarrow 0$ at $872.23 \mathrm{~nm}$, and $2 \rightarrow 0$ at $775.32 \mathrm{~nm}$ rovibrational band spectra. Excellent agreement between values inferred from each band $[(499 \pm 29) \mathrm{K}]$ as well as inferred by using the $3 \rightarrow 0$ band peak ratio method $[(473 \pm 25) \mathrm{K}]$ was obtained for a $600 \mathrm{~W}, 850 \mathrm{G}$, and 10 mTorr helicon $\mathrm{N}_{2}$ plasma. The vibrational temperature was determined from a Boltzmann plot of the integrated band intensities $[(6040 \pm 225) \mathrm{K}]$ and by numerical fit to the $\Delta v=+1,+2$, and +3 sequences after accounting for Franck-Condon factors and electronic-vibrational transition moments $[(6200 \pm 400) \mathrm{K}]$. (C) 2006 American Institute of Physics. [DOI: 10.1063/1.2219392]
\end{abstract}

\section{INTRODUCTION}

Due to its simplicity, high sensitivity, and noninvasive character, optical emission spectroscopy (OES) is the most common way to determine vibrational and rotational distribution functions of electronically excited states in molecular plasmas. Vibrational temperature can provide insights about the relative rates of vibration-vibration and vibrationtranslation energy exchange processes. More importantly, under certain circumstances, ${ }^{1}$ rotational temperature can be a good indicator of the gas temperature, one of the key parameters in driving the reaction rates of many processes. In the case of nitrogen plasmas and/or nitrogen containing plasmas, typically Boltzmann plots or fits of the band envelopes of different bands of the first negative system and/or second positive system are used. Although the first positive system $\left(B{ }^{3} \Pi_{g} \rightarrow A^{3} \Sigma_{u}^{+}\right)$has characteristics that make it a more reliable tool for gas temperature estimation (low excitation threshold, long radiative lifetimes, higher predissociation level, and high emission intensity) $)^{2}$ its complex structure ${ }^{3}$ has hampered its use in OES investigations.

In this work we report on a method of rotational and vibrational temperature determination involving comparison of measurements with numerical simulation of the first positive system $\left(1^{+}\right)$emission spectrum. The computed rotational line positions and intensities are based on published values of the nitrogen molecular constants.

\section{EXPERIMENTAL APPARATUS}

Nitrogen plasma was produced in the steady state, high density, helicon plasma source Compact HElicon waves and instabilities experiment (CHEWIE) described in detail elsewhere. ${ }^{2}$ Briefly, it consists of a $12 \mathrm{~cm}$ long, $6 \mathrm{~cm}$ in di-

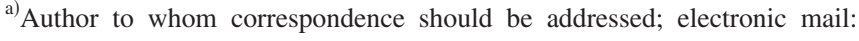
costel.biloiu@mail.wvu.edu
}

ameter Pyrex tube helicon source connected to a $30 \mathrm{~cm}$ long, $15 \mathrm{~cm}$ in diameter stainless steel diffusion chamber. Constant pressure (10-200 mTorr) nitrogen at variable gas flow rate [1-300 SCCM (standard cubic centimeter per minute)] is maintained by a diffusion-rotary vacuum pumping system and calibrated needle valves. A water-cooled electromagnet surrounds the source and is capable of generating a uniform axial magnetic field of $850 \mathrm{G}$ in the source that rapidly diverges to nearly $0 \mathrm{G}$ at the end of the expansion chamber. rf power of up to $600 \mathrm{~W}$ is coupled into the plasma through a $\Pi$-type matching network and a $7 \mathrm{~cm}$ long, water cooled Boswell saddle antenna. For these experiments the driving frequency was chosen $10.75 \mathrm{MHz}$ to match the lower hybrid frequency $f_{\mathrm{LH}} \cong\left(f_{\mathrm{ce}} f_{\mathrm{ci}}\right)^{1 / 2}\left(f_{\mathrm{ce}}\right.$ and $f_{\mathrm{ci}}$ are the electron and ion cyclotron frequencies, respectively) of the approximately $5 \%$ dissociated $\mathrm{N}_{2}$ gas. As the power is increased, the discharge jumps from the weak luminosity capacitively coupled regime ( $E$ mode) into the bright inductively coupled ( $H$ mode) regime. No helicon regime ( $W$ mode) could be attained due to the limited available rf power. Typical electron densities and electron temperatures in $E$ and $H$ modes are: $n_{e} \approx 3 \times 10^{10} \mathrm{~cm}^{-3}$ and $T_{e} \sim 10 \mathrm{eV}$, and $n_{e} \approx 8 \times 10^{11} \mathrm{~cm}^{-3}$ and $T_{e} \sim 5 \mathrm{eV}$, respectively, as measured with a rf compensated Langmuir probe ${ }^{4} 10 \mathrm{~cm}$ downstream in the diffusion region.

Collected light from the plasma was dispersed with a $1.33 \mathrm{~m}$ Czerny-Turner double pass scanning monochromator with a $120 \times 140 \mathrm{~mm}^{2}$ grating having 1200 grooves $/ \mathrm{mm}$ blazed at $750 \mathrm{~nm}$. The linear dispersion of this system is $0.62 \mathrm{~nm} / \mathrm{mm}$ and the maximum resolution is $0.015 \mathrm{~nm}$. Scanning over the range of $500-1100 \mathrm{~nm}$ is accomplished with a computer controlled stepper drive. The detector is an air-cooled SBIG ST-7XEAI dual autofocusing chargecoupled device $(\mathrm{CCD})$ camera having a $765 \times 510$ pixel array at $9 \mu \mathrm{m} /$ pixel. The quantum efficiency is 0.85 at $650 \mathrm{~nm}, \sim 0.45$ toward the blue $(400 \mathrm{~nm})$ and $\sim 0.05$ toward 


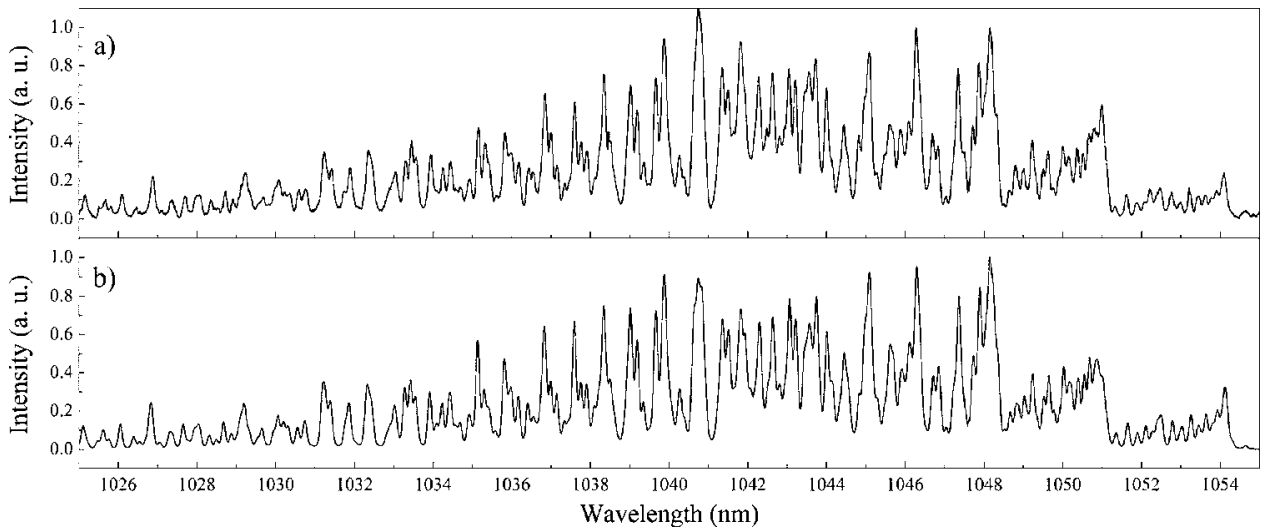

FIG. 1. Measured spectrum (a) and corresponding numerically generated spectrum (b) of the $0-0$ band of $\mathrm{N}_{2}$ for the best fit (502 \pm 29 ) K rotational temperature.

the near infrared $(1000 \mathrm{~nm})$. The acquisition time of the camera ranges from $10^{-2}$ to several hundred seconds. The camera has a high speed interface for PC data acquisition allowing transfer speeds of 1 frame/s. The light emitted by the helicon plasma source was collected from the vicinity of the antenna along the axis of the discharge. The collection optics focused the plasma light into a $200 \mu \mathrm{m}$ core multimode optical fiber that transported the light to the entrance slit of the monochromator. The width of the entrance slit of the spectrometer was set to $80 \mu \mathrm{m}$, and the integration time was short enough $(90 \mathrm{~s})$ that none of the emission bands saturated the detector, but long enough to measure the 0-0 band in the near infrared where the quantum efficiency of the CCD camera is poor. The entire optical path was calibrated in relative spectral response with a tungsten ribbon lamp. Since the spectral window of the spectrometer-CCD camera system spans only $3.5 \mathrm{~nm}$, the entire spectrum of each band was obtained through successive measurements in steps of $2 \mathrm{~nm}$. The $1.5 \mathrm{~nm}$ overlap of adjacent measurements aided reconstruction of each band spectrum. The entire sequence was then assembled after correcting for the wavelength dependent linear dispersion of the spectrometer. Over the $80 \mathrm{~min}$ required to acquire the spectrum of the widest sequence reported here, $\Delta v=+1(789-891 \mathrm{~nm})$, no variations in the plasma parameters were observed.

\section{ROTATIONAL AND VIBRATIONAL TEMPERATURE DETERMINATION}

Simulation of a $\mathrm{N}_{2}$ rovibrational band spectrum requires the knowledge of the individual rotational line positions and intensities. If a whole sequence is to be simulated, the relative intensities of the bands are also required, which in turn requires knowledge of the Franck-Condon factors and electronic-vibrational transition moments or Einstein transition probabilities of the particular vibrational transitions. To calculate the line positions of the nitrogen $1^{+}$band system we used Budo's triplet term formulas, ${ }^{5}$

$$
\begin{aligned}
& F_{J-1, J+1}(J)= \frac{1}{\lambda_{v^{\prime} v^{\prime \prime}}}+B_{v^{\prime}}\left\{-\Lambda^{2}+J(J+1)\right. \\
& \mp \sqrt{y_{1}+4 J(J+1)} \\
&\left.+\frac{2}{3}\left[1-\frac{y_{2}-2 J(J+1)}{y_{1}+4 J(J+1)}\right]+\cdots\right\}, \\
& F_{J}(J)=\frac{1}{\lambda_{v^{\prime} v^{\prime \prime}}}+B_{v^{\prime}}\left\{-\Lambda^{2}+J(J+1)\right. \\
&\left.+\frac{2}{3}\left[1+2 \frac{y_{2}-2 J(J+1)}{y_{1}+4 J(J+1)}\right]+\cdots\right\},
\end{aligned}
$$

where $1 / \lambda_{v^{\prime} v^{\prime \prime}}$ is the energy difference between upper $\left(v^{\prime}\right)$ and lower $\left(v^{\prime \prime}\right)$ vibrational levels, $B_{v^{\prime}}$ and $J$ are the rotational constant and rotational quantum number, respectively, and $y_{1}=\Lambda^{2} Y(Y-4)+4 / 3$ and $y_{2}=\Lambda^{2} Y(Y-1)-4 / 9$ are two parameters that depend on the orbital quantum number $(\Lambda)$ and on the ratio of the spin-orbit constant to the rotational constant $\left(Y=\mathrm{A}_{v^{\prime}} / B_{v^{\prime}}\right)$.

Under the assumptions of Hund's case (a) for the upper $B{ }^{3} \Pi_{g}$ electronic state, ${ }^{6}$ the intensity of an emitted rotational line belonging to any band of the $1^{+}$system is (see Ref. 2 and references therein for a detailed discussion on line intensity),

$$
\begin{aligned}
& I_{A v^{\prime \prime} J^{\prime \prime}}^{B v^{\prime} J^{\prime}}=\text { const } \times \varepsilon(\lambda) A_{v^{\prime} v^{\prime \prime}} \exp \left[-\left(h c G\left(v^{\prime}\right) / k_{B} T_{v}\right)\right] \\
& \times\left(\Phi_{J^{\prime}} S_{J^{\prime} J^{\prime \prime}} / T_{r} \lambda\right) \exp \left\{-\left(h c / k_{B} T_{r}\right)\right. \\
& \left.\times\left[\mathrm{A}_{v^{\prime}} \Lambda \Sigma+B_{v^{\prime}} J^{\prime}\left(J^{\prime}+1\right)\right]\right\},
\end{aligned}
$$

where $\varepsilon(\lambda)$ is the overall optical path sensitivity (windows, collection optics, optical fiber, spectrometer, and CCD camera), $A_{v^{\prime} v^{\prime \prime}}=\left(64 \pi^{4} / 3 h \lambda^{3}\right) q_{v^{\prime} v^{\prime \prime}}\left|R_{e}\left(r_{v^{\prime} v^{\prime \prime}}\right)\right|^{2}$ is the band transition probability (with $q_{v^{\prime} v^{\prime \prime}}$ and $\left|R_{e}\left(r_{v^{\prime} v^{\prime \prime}}\right)\right|^{2}$, the FranckCondon factor and electronic-vibrational transition moment, respectively), $G\left(v^{\prime}\right)=\Sigma_{i} \alpha_{i}\left(v^{\prime}+\frac{1}{2}\right)^{i}$ is the vibrational energy term (with $\alpha_{i}$ the equilibrium molecular constants), $h$ and $k_{B}$ are the Planck and Boltzmann constants, $T_{v}$ and $T_{r}$ are the vibrational and rotational temperatures, respectively, $c$ is the speed of light, $\Phi_{J^{\prime}}$ is the $1: 2$ intensity alternation factor, $S_{J^{\prime} J^{\prime \prime}}$ is the line strength, and $\Sigma$ is the Hund's case (a) quantum number (the component of the spin along the internu- 


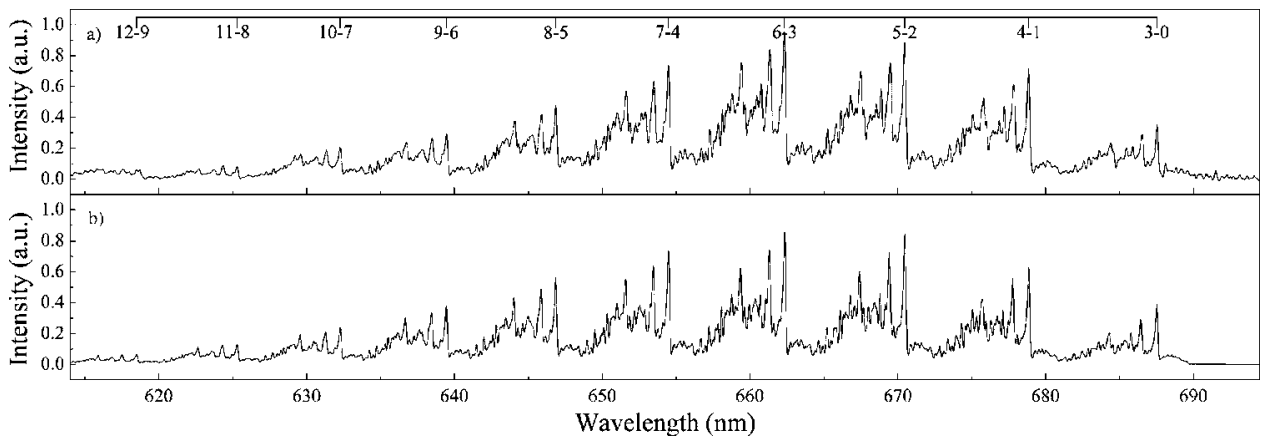

FIG. 2. Experimental spectrum (a) and corresponding synthetically generated spectrum (b) of the $\Delta v=+3$ band sequence for the best fit (6180 \pm 370 ) K vibrational temperature.

clear axis). For all the energy terms in $\mathrm{cm}^{-1}$ and $h c / k_{B}=1.44 \mathrm{~cm} \mathrm{~K}$, the temperatures in Eq. (2) are in Kelvin.

To compare the simulated spectrum to a measured spectrum, the simulated spectrum must be convoluted with the apparatus function of the spectrometer. The wavelength dependent analytical form of the apparatus function was determined by a fit of the measured atomic lines of a low temperature Ar lamp with a pseudo-Voight function,

$$
f_{\text {app }}(\lambda)=p(\lambda) f_{G}(\lambda)+[1-p(\lambda)] f_{L}(\lambda),
$$

where $p$ and $1-p$ are the relative magnitudes of the Gauss and Lorentz function contributions, respectively. The resultant simulated spectrum is then fitted to the measured spectrum using a nonlinear fitting algorithm to obtain the best fit values of $T_{r}$ and $T_{v}{ }^{7}$ Although reliable, the nonlinear fitting procedure is time intensive. To fit the simulated 32000 points of the $\Delta v=+2$ sequence to the measured spectrum required $5 \mathrm{~h}$ of computational time on a Pentium $\mathrm{V}^{\mathrm{TM}}$ processor. To speed the analysis procedure, the rotational and vibrational temperatures can be determined sequentially, and then the entire simulated spectrum compared to the measured spectrum. The rotational line positions given by Eq. (1) are sufficient for use in a simulation of the entire sequence. However, more precise line positions are needed for simulation of an individual rotational band. By taking only the rotation dependent part of Eq. (2) and using high precision $\left( \pm 0.007 \mathrm{~cm}^{-1}\right)$ measured line positions ${ }^{8}$ the rotational temperature (effectively the gas temperature) has been inferred from individual fits of the $0-0,1-0$, and 2-0 rotational bands. To simulate the individual rotational bands, we included all 27 branches $(P, Q, R)_{i j}$, with $i, j=1,2,3$ and $J$ values as high as 40. The Hönl-London factors were computed from the formulas given by Kovacs ${ }^{9}$ for triplet transitions in the intermediate regime between Hund's (a) and (b) cases. The relevant constants were calculated from the equilibrium molecular constants of the $B{ }^{3} \Pi_{g}$ and $A^{3} \Sigma_{u}{ }^{+}$states. ${ }^{10}$ As shown in Fig. 1, an excellent reproduction of the measured spectra is obtained; the small differences in intensities that appear between the numerically generated spectrum and measured spectrum are due to the change in spin-orbit constant as $J^{\prime}$ increases and the $B{ }^{3} \Pi_{g}$ state changes its character from Hund's case (a) toward case (b). For 10 mTorr, $850 \mathrm{G}$ and $600 \mathrm{~W}$ input power, the $T_{r}$ values obtained from these bands $[(502 \pm 29) \mathrm{K},(508 \pm 29) \mathrm{K},(486 \pm 28) \mathrm{K}$ for $0-0,1-0$, and 2-0 bands, respectively] were in excellent agreement with each other (within 6\%) and with the temperature inferred by the peak ratio method $[(473 \pm 25) \mathrm{K}]$ using the $3-0$ band. ${ }^{11}$ Once $T_{r}$ is determined, an entire band sequence can be modeled by adding the vibration dependent portion of Eq. (2) back into the model. An example of a generated rovibrational spectrum for the $\Delta v=+3$ sequence is shown in Fig. 2. Again, the nonlinear fitting process used to determine the best fit value of $T_{v}$ is time consuming. Therefore, it is quicker to determine $T_{v}$ from the Boltzmann plot, ln $\left(I_{v^{\prime} v^{\prime \prime}} \varepsilon(\lambda) \lambda_{v^{\prime} v^{\prime \prime}} / A_{v^{\prime} v^{\prime \prime}}\right)$ vs $G\left(v^{\prime}\right)$ [with $I_{v^{\prime} v^{\prime \prime}} \varepsilon(\lambda)$ and $\lambda_{v^{\prime} v^{\prime \prime}}$, the integrated band intensities corrected for spectral sensitivity and band origin wavelength, respectively] (see Fig. 3). The errors introduced into the analysis by incomplete separation of the partially overlapping adjacent bands are smaller than the errors due to uncertainties in the band transition probabilities $^{12}$ or Franck-Condon factors and transition moment values ${ }^{13,14}$ except for the weakest detected bands in each sequence. From the Boltzmann plot, a vibrational temperature of $T_{v}=6040 \pm 225 \mathrm{~K}$ is obtained. This value is also in agreement with the average value of $(6200 \pm 400) \mathrm{K}$ obtained by independent nonlinear fit of the $\Delta v=+1,+2$, and +3 sequences. The substantial difference between $T_{r}$ and $T_{v}$ is normal for the discharge type and working conditions employed. Because of the small energy separation of the rotational levels, in low temperature plasmas almost all gas kinetic collisions produce a change in the rotational quantum number, and relatively short rotational relaxation times re-

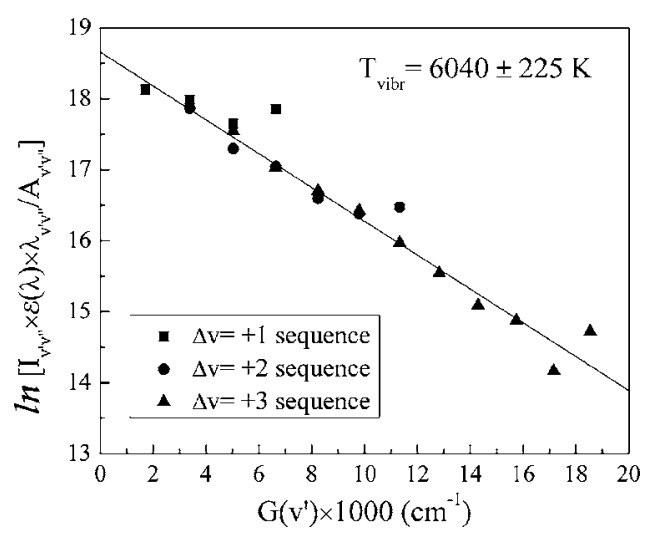

FIG. 3. Vibrational temperature determination from the Boltzmann plot of the $\Delta v=+1,+2$, and +3 sequences. The four-points farthest from the line were not used in the fit; they correspond to the lowest intensity bands and therefore lack the precision of the other measurements. 
sult. Thus, rotational distributions can equilibrate with the gas kinetic temperature. However, collisions able to produce a change in the vibrational quantum number are much less frequent, and therefore the vibrational distribution is not expected to reach equilibrium with translational and/or rotational degrees of freedom.

The method presented here allows accurate determination of the rotational and vibrational temperatures of $\mathrm{N}_{2}$ molecules. For nitrogen plasmas in which thermal equilibrium between rotational and translational degrees of freedom of the gas molecules is attained, ${ }^{2}$ the method can be used as a sensitive "thermometer" for the gas temperature. Even for plasmas that do not normally contain $\mathrm{N}_{2}$, trace amounts of $\mathrm{N}_{2}$ can be used as a molecular actinometer and the gas temperature determined spectroscopically.

${ }^{1}$ B. A. Cruden, M. V. Rao, S. P. Sharma, and M. Meyyappan, J. Appl. Phys. 91, 8955 (2002).
${ }^{2}$ C. Biloiu, X. Sun, Z. Harvey, and E. Scime, J. Appl. Phys. (submitted).

${ }^{3}$ A. Lofthus and P. Krupenie, J. Phys. Chem. Ref. Data 6, 115 (1977).

${ }^{4}$ C. Biloiu, E. Scime, X. Sun, and B. McGeehan, Rev. Sci. Instrum. 75, 4296 (2004)

${ }^{5}$ A. Budo, Z. Phys. 107, 73 (1937).

${ }^{6}$ G. Herzberg, Spectra of Diatomic Molecules, Molecular Spectra and Molecular Structure Vol. I (Krieger, Malabar, FL, 1989), p. 219.

${ }^{7}$ H. Nassar, S. Pellerin, K. Musiol, O. Martinie, N. Pellerin, and J. M. Cormier, J. Phys. D 37, 1904 (2004).

${ }^{8}$ C. Effantin, C. Amiot, and J. Verges, J. Mol. Spectrosc. 76, 221 (1979).

${ }^{9}$ I. Kovacs, Rotational Structure in the Spectra of Diatomic Molecules (American Elsevier, New York, 1969), p. 136.

${ }^{10}$ F. Roux, F. Michaud, and J. Verges, J. Mol. Spectrosc. 97, 253 (1983).

${ }^{11}$ M. Simek and S. De Benedictis, Plasma Chem. Plasma Process. 15, 451 (1995).

${ }^{12}$ L. G. Piper, K. W. Holtzclaw, B. D. Green, and W. A. M. Blumberg, J. Chem. Phys. 90, 5337 (1989).

${ }^{13}$ C. O. Laux and C. H. Kruger, J. Quant. Spectrosc. Radiat. Transf. 48, 9 (1992).

${ }^{14}$ F. R. Gilmore, R. R. Laher, and P. J. Espy, J. Phys. Chem. Ref. Data 21, 1005 (1992). 